\title{
Plasticidade fenotípica de Baccharis genistelloides subsp. crispa (Spreng.) Joch. Müll. (2006) - Asteraceae - sob manejo orgânico
}

SOUSA, L.A. ${ }^{1}$; GRATÃO, A.S. ${ }^{1}$; LEITE, M.N..; ANDRADE, F.M.C..'; CECON, P.R..'; FREITAS, G.B. ${ }^{1}$ ${ }^{1}$ Departamento de Fitotecnia da Universidade Federal de Viçosa, Avenida Peter Henry Rolfs, s/n, Campus Universitário, Viçosa, MG, CEP 36570-000, TEL. (31) 3899-1346; '²Departamento de Farmácia, Laboratório de Farmacognosia da Universidade Federal de Juiz de Fora

RESUMO: O objetivo deste trabalho, relacionado a produção orgânica, utilizou preparados homeopáticos visando incremento na biomassa e no teor de flavonóides em plantas dióicas de carqueja. Os tratamentos foram: controle com água, controle com álcool $70 \%$, Phosphorus $12 \mathrm{CH}$, Sulphur $6 \mathrm{CH}$, e a combinação destas homeopatias. Variações fenotípicas foram registradas a partir da análise de crescimento, realizada quinzenalmente durante 60 dias e dos caracteres morfológicos, tais como: como altura, número de ramificações, comprimento do ramo principal, diâmetro do caule e ala caulinar mediana. Na colheita foram medidos: a fitomassa fresca/seca e o volume de raiz. Posteriormente, foi quantificado o teor de flavonóides totais conforme as análises prescritas pela Farmacopéia Brasileira. O experimento foi avaliado em delineamento de blocos casualizados ( $2 \times 5 \times 2)$, sendo suas médias avaliadas pelo teste Tukey e o coeficiente de variação ambiental para estimar a plasticidade. Quanto ao estudo do crescimento da planta ao longo do tempo, verificou-se aumento linear simples para a maioria das variáveis nos dois sexos. Foram detectadas respostas plásticas na maioria dos caracteres avaliados. De acordo com os resultados, em ambos os sexos, 60 dias de cultivo são suficientes para obtenção de matéria prima com teor aceitável de flavonóides, bem como para verificação dos efeitos de patogenesia e similitude na aplicação de preparados homeopáticos Phosphorus 12CH, Sulphur $6 \mathrm{CH}$ e sua combinação Phosphorus $12 \mathrm{CH}+$ Sulphur $6 \mathrm{CH}$. A fitomassa e o teor de flavonóides totais foram semelhantes entre plantas dióicas durante o período de estudo.

Palavras-chave: agroecologia, biomassa, fitotecnia, flavonóide, homeopatia vegetal.

ABSTRACT: Phenotypic plasticity of Baccharis genistelloides subsp. crispa (Spreng.) Joch. Müll. (2006) - Asteraceae - under organic management. This study looked at organic production, in addition to the use of homeopathic preparations, in order to increase biomass and flavonoid content in dioecious plants of Baccharis genistelloides. The adopted treatments were: water control, control with $70 \%$ alcohol, Phosphorus $12 \mathrm{CH}$, Sulphur $6 \mathrm{CH}$ and a combination of them. Phenotypic changes were recorded for the growth analysis, performed biweekly for 60 days, of the morphological features, such as height, number of branches, length of the main branch, diameter of the stem and median stem wing. At harvest, we measured the fresh/dry phytomass and root volume. Subsequently, we quantified the content of total flavonoids according to the analyses required by the Brazilian Pharmacopoeia. The experiment was evaluated in a randomized block design, and its averages were evaluated by the Tukey's test and environmental variation coefficient to estimate plasticity. As for the study of plant growth over time, there was a simple linear increase for most of the variables in both genders. We could detect phenotypic plasticity traits in most features analyzed. According to these results in both genders, 60 days after inoculation are sufficient to obtain raw material with acceptable level of flavonoids, as well as pathogenesis and similar effects in the application of homeopathic preparations of Phosphorus $12 \mathrm{CH}$, Sulphur $6 \mathrm{CH}$ and their combination. Phytomass and content of total flavonoids were similar between the dioecious plants during the study period.

Keywords: agroecology, biomass, phytotechny, flavonoid, plant homeopathy. 


\section{INTRODUÇÃO}

A carqueja, Baccharis genistelloides subsp. crispa (Asteraceae), é uma planta com propriedades medicinais e aromáticas (Vieira et al., 2002) de ampla distribuição nacional, ocorrendo pelos biomas do Cerrado (Rodrigues \& Carvalho, 2001), Mata Atlântica (Pavan-Fruehauf, 2000; Vieira et al., 2002) e Pampa (Ritter \& Baptista, 2005; Caporal \& Boldrini, 2007). Habita ambientes ruderais (Vieira et al., 2002; Brandão \& Oliveira 1995; Macedo, 1995), sendo ainda adotada como espécie bioindicadora de solos compactados (Burg \& Mayer, 2002).

Os estudos dos processos de interação planta-ambiente são indispensáveis ao entendimento do mecanismo de adaptação de espécies, quando submetidas a diferentes condições de cultivo (Montanari et al., 2004) e, nas últimas décadas, o conhecimento sobre a plasticidade fenotípica tem progredido significativamente (Pigliucci, 2006).

A plasticidade fenotípica confere vantagem seletiva crucial às plantas, por constituir uma base epigenética para o melhoramento vegetal (Gottlieb et al., 1996). Desta forma, Clement (2001) considera o melhoramento genético como o principal processo que transforma algum componente da biodiversidade em recurso genético e, finalmente, em produto com valor econômico no mercado moderno.

Estudos envolvendo a utilização de homeopatia no cultivo de plantas medicinais revelam aplicações viáveis. Bonfim et al. (2008), por exemplo, verificaram que preparados homeopáticos de Arnica montana $3 \mathrm{CH}, 6 \mathrm{CH}$ e $12 \mathrm{CH}$ promoveram aumento na porcentagem e qualidade de raízes na estaquia de Lippia alba; Carvalho et al. (2005), avaliando o efeito dos mesmos preparados nas potências de $\mathrm{CH} 3$ e $\mathrm{CH} 5$ sobre plantas de artemísia relatam que o teor de partenolídeo diminuiu. Bonato et al. (2009) sugerem que os medicamentos Sulphur e Arsenicum album modificam o metabolismo de Mentha arvensis, especialmente aumentando o teor de metabólitos secundários como o óleo essencial. Andrade (2000) verificou que chambá (Justicia pectorailis) respondeu aos preparados Phosphorus e Sulphur, ambos $\mathrm{CH} 3$, aumentando o teor de cumarina comparados às testemunhas, apresentando alteração no campo eletromagnético conforme revelado pela técnica de fotos Kirlian.

O uso da homeopatia na agricultura ainda apresenta lacunas, principalmente, quando o objetivo é equilibrar os agroecossistemas e ampliar o potencial produtivo das plantas. Entretanto, Carvalho et al. (2005) e Andrade et al. (2001) sugerem que a Homeopatia seja alternativa compatível com as visões orgânica, holística, sistêmica e ecológica, que utilizam preparados que estimulam as defesas naturais dos organismos.

O objetivo desta pesquisa consistiu em estabelecer um sistema de produção orgânica com uso de preparados homeopáticos utilizando Phosphorus e Sulphur em diferentes concentrações, com vistas ao incremento de biomassa e da qualidade quanto ao teor de flavonóides totais, expresso em quercetina, em genótipos masculinos e femininos de $B$. genistelloides subsp. crispa.

\section{MATERIAL E MÉTODOS}

A pesquisa foi desenvolvida durante os meses de setembro a dezembro de 2010 no Setor de Agroecologia da Universidade Federal de Viçosa, localizada no município de Viçosa-MG, situado a $20^{\circ} 45^{\prime} 25,2^{\prime \prime S}$ e $42^{\circ} 52^{\prime} 09,5^{\prime \prime} \mathrm{O}$, na altitude média de 652 m. Conforme Köppen e Greiger (1928), o clima é classificado por Cwa.

O material botânico foi coletado e submetido à confecção de exsicatas, sendo posteriormente incorporado ao acervo do Herbário do Departamento de Biologia Vegetal/UFV (VIC 34.431 para o genótipo masculino e VIC 34.433 para o genótipo feminino).

Inicialmente, foram obtidas estacas (clones) de plantas de carqueja femininas (25) e masculinas (25), em processo incipientemente de domesticação, subcategorizado conforme Clement (2001). As estacas eram procedentes de mudas coletadas em barrancos de beiras de estradas que dão acesso à UFV e, à posteriori, foram manejadas para obtenção de ramos caulinares cultivados em sacos plásticos de $4 \mathrm{~L}$ utilizando-se como substrato esterco bovino curtido e solo de barranco (1:2), ou seja, 33,5t/ha de adubo orgânico e espaçamento de $0,25 \times 0,25 \mathrm{~cm}$. $\mathrm{Na}$ Tabela 1 encontra-se a análise físico-química

TABELA 1. Características físico-químicas de amostra do solo de barranco coletadas, no município de ViçosaMG em 2010

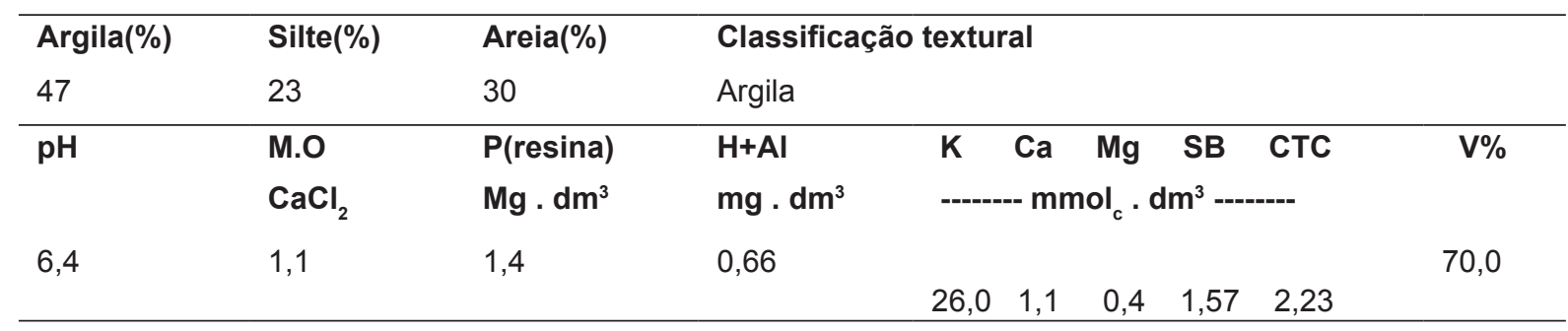

Rev. Bras. PI. Med., Campinas, v.16, n.2, p.231-236, 2014. 
de amostras do solo de barranco utilizado nos experimentos.

Os tratamentos com preparados homeopáticos foram: controle/testemunha com água, controle/testemunha com etanol $70 \%$, preparado homeopático Phosphorus $12 \mathrm{CH}$, preparado homeopático Sulphur $6 \mathrm{CH}$ e a combinação dos preparados homeopáticos Phosphorus $12 \mathrm{CH}+$ Sulphur $6 \mathrm{CH}$ para os dois sexos. Os preparados homeopáticos foram adquiridos no Laboratório de Homeopatia do Departamento de Fitotecnia/UFV.

As variações fenotípicas foram registradas quinzenalmente durante 60 dias com auxílio de paquímetro e trena. Foram medidos os seguintes caracteres morfológicos: altura (ALT-cm), número de ramificações/alas caulinares (NRAM), comprimento do ramo principal (CRP-cm), diâmetro do caule (DCA-mm) e ala caulinar mediana (ALAM-cm). Em pós colheita foi avaliado a fitomassa fresca/ seca com auxílio de balança digital (PF/PS-g), o volume de raiz (VR-mL) com auxílio de proveta, e o teor de flavonóides (FLA) totais (\%). A análise do teor de flavonóides foi realizada em fevereiro de 2011, no Laboratório de Farmacognosia e Núcleo de Identificação Química e Analítica da Universidade Federal de Juiz de Fora. As amostras foram submetidas à quantificação quanto ao teor de flavonóides totais, calculados como quercetina (Farmacopéia Brasileira, 2002).

O experimento foi instalado em esquema fatorial $2 \times 5$, considerando dois sexos e cinco tratamentos de adubação em delineamento em blocos casualizados, com cinco repetições. Os dados foram analisados por meio da análise de variância e de regressão. As médias das amostras entre os fatores sexos e tratamentos foram comparadas utilizando-se o teste Tukey a $5 \%$ e o fator quantitativo dos modelos foi escolhido conforme a significância dos coeficientes de regressão e no coeficiente de determinação, incluindo o coeficiente de variação ambiental (CVa), visando estimar a intensidade da resposta plástica. Os dados foram processados no SAEG (2007).

\section{RESULTADOS E DISCUSSÕES}

Os resultados encontrados nas amostras de plantas de carqueja, de ambos os sexos, demonstraram que 60 dias de cultivo, utilizando solo com as características físico-químicas descritas na Tabela 1 , são suficientes para a obtenção de matéria prima com qualidade para a produção de fitoterápicos, estimando-se 1.327,16 Kg/ha de matéria seca e $7,12 \mathrm{Kg} / \mathrm{ha}$ de flavonóides totais.

Verificou-se que houve efeito significativo dos tratamentos de adubação orgânica com seus complementos à base de preparados homeopáticos aos 60 dias para alguns caracteres, conforme as avaliações finais registradas nas Tabelas 2 e 4 . A altura das plantas (ALT cm) não diferiu nas plantas femininas entre os tratamentos e controles (Tabela 2). Entretanto, nas plantas masculinas, as plantas submetidas aos tratamentos homeopáticos foram significativamente menores do que as do controle, exceto para aquelas tratadas com Sulphur $\mathrm{CH} 6$ (Tabela 2). A altura das plantas entre os sexos diferiu apenas no tratamento Phosphorus $12 \mathrm{CH}$, em que machos foram menores, e na testemunha álcool, em que as plantas femininas foram menores do que as masculinas (Tabela 2).

Com relação ao número de ramificações (NRAM) foram registrados maiores valores para plantas femininas nos tratamentos Phosphorus $12 \mathrm{CH}$ e testemunha água, porém não diferiram significativamente (Tabela 2 ). O número de ramificações foi maior para plantas masculinas na testemunha álcool e Sulphur $6 \mathrm{CH}$ e não diferiu no tratamento Phosphorus $12 \mathrm{CH}+$ Sulphur $6 \mathrm{CH}$ (Tabela 2).

Para o diâmetro do caule (DCA) não houve efeito entre tratamentos para plantas de ambos os sexos, mas a testemunha álcool e a combinação Phosphorus $12 \mathrm{CH}+$ Sulphur $6 \mathrm{CH}$ reduziram o diâmetro de plantas femininas (Tabela 2). Com relação ao comprimento do ramo principal (CRP), não houve diferença (Tabela 2). Quanto à ala caulinar mediana (ALAM) também não houve efeito significativo dos tratamentos, porém as plantas masculinas apresentaram maior largura caulinar $(\mathrm{cm})$ em relação às plantas femininas (Tabela 2).

Não houve diferença entre as plantas femininas e masculinas quanto à produção de fitomassa fresca (FF), seca (FS) e quanto ao teor de flavonóides (\%) dentro de cada tratamento (Tabela 3 ). Entretanto, as plantas masculinas produziram raízes com maior volume em relação às femininas, independente do tratamento (Tabela 3).

Quanto ao desenvolvimento ao longo do tempo verificou-se efeito linear simples na maioria das variáveis nos dois sexos. Foi possível detectar resposta plástica para a maioria dos caracteres através do Coeficiente de Variação Ambiental (Tabelas 2 e 3 ).

O efeito notório, também verificado em outras pesquisas agronômicas (Castro \& Ferreira, 2000; Silva, 2001; Sousa, 2005) no cultivo de carquejas, demonstrara que a utilização de cerca de $30 \mathrm{t} / \mathrm{ha}$ de esterco bovino curtido é uma dose compatível para a produtividade de metabólitos secundários, considerando-se que os genótipos femininos e masculinos produziram teores aceitáveis de flavonóides, conforme preconizado pela Farmacopéia Brasileira (2002).

Quanto ao resultado encontrado para o

Rev. Bras. PI. Med., Campinas, v.16, n.2, p.231-236, 2014. 
TABELA 2. Média dos caracteres morfológicos (ALT - altura; NRAM - número de ramificações; CRP comprimento do ramo principal; DCA - diâmetro do caule; ALAM - ala caulinar mediana; Cva - coeficiente de variação ambiental) avaliados nos tratamentos de adubação orgânica e seus complementos com preparados homeopáticos (T): Phosphorus $12 \mathrm{CH}(\mathrm{Ph})$, Testemunha água (Ta), Testemunha etanol (Te), Phosphorus $12 \mathrm{CH}+$ Sulphur $6 \mathrm{CH}$ (PS) e Sulphur $6 \mathrm{CH}$ (Su) entre os genótipos masculino ( ${ }^{\Uparrow}$ ) e feminino () de Baccharis genistelloides subsp. crispa após 60 dias de cultivo, no município de Viçosa-MG durante 2010

\begin{tabular}{|c|c|c|c|c|c|c|c|c|c|c|}
\hline \multirow{2}{*}{$\mathrm{T}$} & \multicolumn{2}{|c|}{ ALT (cm) } & \multicolumn{2}{|l|}{ NRAM } & \multicolumn{2}{|c|}{ CRP (cm) } & \multicolumn{2}{|c|}{$\mathrm{DCA}(\mathrm{mm})$} & \multicolumn{2}{|c|}{ ALAM (cm) } \\
\hline & $\hat{\jmath}$ & 우 & $\hat{0}$ & q & $\hat{0}$ & 우 & $\hat{0}$ & 우 & $\hat{0}$ & q \\
\hline $\mathrm{Ph}$ & $47,80^{\mathrm{Bb}}$ & $63,20^{\mathrm{Aa}}$ & $13,40^{\mathrm{Ab}}$ & $21,20^{\mathrm{ABa}}$ & $78,20^{\mathrm{Aa}}$ & $69,40^{\mathrm{Aa}}$ & $4,20^{\mathrm{Aa}}$ & $3,60^{A a}$ & $48,06^{\mathrm{Aa}}$ & $33,72^{\mathrm{Ab}}$ \\
\hline $\mathrm{Ta}$ & $63,33^{\mathrm{Aba}}$ & $58,80^{\mathrm{Aa}}$ & $13,60^{A b}$ & $22,20^{\mathrm{Aa}}$ & $70,60^{\mathrm{Aa}}$ & $72,20^{\mathrm{Aa}}$ & $4,0^{\mathrm{Aa}}$ & $3,60^{\mathrm{Aa}}$ & $43,79^{A a}$ & $35,20^{A b}$ \\
\hline $\mathrm{Te}$ & $72,0^{\mathrm{Aa}}$ & $45,0^{A b}$ & $13,20^{\mathrm{Ab}}$ & $7,80^{\mathrm{ABa}}$ & $79,80^{\text {Аa }}$ & $68,0^{\mathrm{Aa}}$ & $4,0^{\mathrm{Aa}}$ & $3,20^{A b}$ & $42,93^{\mathrm{Aa}}$ & $34,53^{A b}$ \\
\hline PS & $47,0^{\mathrm{Ba}}$ & $60,0^{A a}$ & $13,20^{\mathrm{Aa}}$ & $16,0^{\mathrm{Ba}}$ & $79,60^{\mathrm{Aa}}$ & $71,90^{\mathrm{Aa}}$ & $4,40^{A a}$ & $3,20^{\mathrm{Ab}}$ & $47,26^{\mathrm{Aa}}$ & $32,33^{A b}$ \\
\hline Su & $62,20^{\mathrm{ABa}}$ & $63,25^{\mathrm{Aa}}$ & $11,60^{\mathrm{Ab}}$ & $7,60^{\mathrm{ABa}}$ & $75,90^{\mathrm{Aa}}$ & $67,0^{\mathrm{Aa}}$ & $4,0^{\mathrm{Aa}}$ & $3,80^{\mathrm{Aa}}$ & $44,19^{A a}$ & $36,39^{A b}$ \\
\hline Cva\% & \multicolumn{2}{|c|}{18,24} & \multicolumn{2}{|l|}{25,08} & \multicolumn{2}{|c|}{10,14} & \multicolumn{2}{|c|}{12,86} & \multicolumn{2}{|l|}{8,58} \\
\hline
\end{tabular}

As médias seguidas de pelo menos uma mesma letra maiúscula na coluna (tratamentos) e minúscula na linha (sexos) não diferem ao nível de $5 \%$ de probabilidade pelo teste Tukey

TABELA 3. Média dos caracteres da Biomassa fresca e seca, Volume de raiz e Flavonóides (expresso em quercetina, \%) avaliados nos tratamentos de adubação orgânica e seus complementos com preparados homeopáticos (T): Phosphorus $12 \mathrm{CH}(\mathrm{Ph})$, Testemunha água (Ta), Testemunha etanol (Te), Phosphorus 12CH + Sulphur 6CH (PS), Sulphur 6CH (Su) e Cva (coeficiente de variação ambiental) entre os sexos masculino e feminino de Baccharis genistelloides subsp. crispa no pós-colheita, DFT/UFV, no município de Viçosa-MG durante 2010

\begin{tabular}{|c|c|c|c|c|c|c|c|c|}
\hline \multirow{2}{*}{$\mathrm{T}$} & \multicolumn{2}{|c|}{ Biomassa fresca (g) } & \multicolumn{2}{|c|}{ Biomassa seca $(\mathrm{g})$} & \multicolumn{2}{|c|}{ Volume de raiz $\left(\mathrm{mL}^{3}\right)$} & \multicolumn{2}{|c|}{ Flavonóides (\%) } \\
\hline & $\hat{0}$ & 우 & $\hat{0}$ & & $\hat{0}$ & 운 & $\hat{0}$ & 우 \\
\hline $\mathrm{Ph}$ & $25,60^{\mathrm{Aa}}$ & $21,12^{\mathrm{Aa}}$ & $9,54^{\mathrm{Aa}}$ & $8,74^{\mathrm{Aa}}$ & $140^{\mathrm{Aa}}$ & $82^{\mathrm{Ab}}$ & $0,55^{\mathrm{Aa}}$ & $0,58^{\mathrm{Aa}}$ \\
\hline $\mathrm{Ta}$ & $22,91^{\mathrm{Aa}}$ & $21,68^{\mathrm{Aa}}$ & $8,70^{\mathrm{Aa}}$ & $7,87^{\mathrm{Aa}}$ & $90^{\mathrm{Aa}}$ & $80^{\mathrm{Bb}}$ & $0,57^{\text {Аa }}$ & $0,62^{\mathrm{Aa}}$ \\
\hline $\mathrm{Te}$ & $21,10^{\mathrm{Aa}}$ & $19,16^{\mathrm{Aa}}$ & $8,03^{\mathrm{Aa}}$ & $6,56^{\mathrm{Aa}}$ & $100^{\mathrm{Aa}}$ & $68^{\mathrm{Bb}}$ & $0,32 \mathrm{Aa}$ & $0,48^{\mathrm{Aa}}$ \\
\hline PS & $27,98^{\mathrm{Aa}}$ & $25,87^{\mathrm{Aa}}$ & $10,17^{\mathrm{Aa}}$ & $7,30^{\mathrm{Aa}}$ & $90^{\mathrm{Aa}}$ & $80^{\mathrm{Bb}}$ & 0,58 Аа & $0,58^{\mathrm{Aa}}$ \\
\hline Su & $23,34^{\mathrm{Aa}}$ & $18,82^{\mathrm{Aa}}$ & $8,79^{A a}$ & $7,21^{\mathrm{Aa}}$ & $100^{\mathrm{Aa}}$ & $82 \mathrm{Bb}$ & $0,51^{\mathrm{Aa}}$ & $0,53^{\mathrm{Aa}}$ \\
\hline Cva\% & 13,14 & & 13,39 & & 21,61 & & 15,82 & \\
\hline
\end{tabular}

As médias seguidas de pelo menos uma mesma letra maiúscula na coluna (tratamentos) e minúscula na linha (sexos) não diferem ao nível de $5 \%$ de probabilidade pelo teste Tukey

equilíbrio entre os organismos sadios de carqueja foi confirmado estatisticamente que houve casos de patogenesia como: Phosphorus $12 \mathrm{CH}$ e a combinação Phosphorus $12 \mathrm{CH}+$ Sulphur $6 \mathrm{CH}$ reduzem a altura para plantas masculinas (Tabela 2), o que também foi observado em Cymbopogon citratus (Casali et al., 2009); porém não interferiu na produção de fitomassa fresca e seca (Tabela 3); em plantas femininas ocorreu redução para o número de ramificação a combinação de Phosphorus $12 \mathrm{CH}$ + Sulphur $6 \mathrm{CH}$, mas quanto à produtividade não houve interferência para estas condições conforme a Tabela 3.

De acordo com as análises estatísticas ainda foi possível detectar padrões de similitude entre os efeitos provocados pelos tratamentos homeopáticos visto que não diferiram significativamente, pois considerando que não houve diferenças com controle/testemunha com água (Tabelas 2 e 3) sugere-se que os genótipos utilizados nesta pesquisa estão equilibrados e apresentam potencial para uso em estudos de melhoramento genético vegetal.

Os valores de CVa inferiores a $10 \%$ indicam baixa magnitude da plasticidade fenotípica, valores entre 10 e $20 \%$ indicam média magnitude e valores superiores a $20 \%$ indicam alta magnitude de plasticidade fenotípica (Schlichting \& Levin, 1984).

Os caracteres NRAM e VR foram os que demonstraram alta magnitude de plasticidade fenotípica (Tabelas 2 e 3 ), indicando que a morfologia da planta possui grande amplitude de respostas às interações de adubação orgânica nas condições de cultivo experimental durante a primavera.

Apesar dos efeitos de Phosphorus $\mathrm{CH}$ 12, Phosphorus e Sulphur $\mathrm{CH} 12 / 6$ e Sulphur $\mathrm{CH}$

Rev. Bras. PI. Med., Campinas, v.16, n.2, p.231-236, 2014. 
6 , recomenda-se novos estudos que confirmem a eficiência destas potências em diferentes estações do ano e colheita durante outras fenofases, pois elas podem representar equilíbrio para a concentração de flavonóides totais durante a primavera e fenofase vegetativa.

Fatores endógenos e exógenos (GoboNeto \& Lopes, 2007) também podem ter contribuído para a expressão do fenótipo pré selecionado nesta pesquisa, além de fortalecer a premissa de que a agricultura orgânica gera uma matéria prima com qualidade, coerente ao proposto por CORREA Jr. \& SCHEFFER (2009), pois atende ao BPA Boas práticas agrícolas para o cultivo de plantas medicinais.

No âmbito da conservação a curto, médio e longo prazo, a pesquisa favorece as leis da natureza conforme Arruda et al. (2005) e Lisboa et al. (2005), bem como corrobora também com o Decreto $\mathrm{N}^{\circ}$ 5.813 , de 22 de junho de 2006, que preconiza o desenvolvimento de sistemas de produção orgânica de plantas medicinais, viabilizando a cadeia produtiva de plantas medicinais nativas e exóticas, auxiliando programas de bioprospecção.

\section{CONCLUSÃO}

Os resultados permitem concluir que, apesar do dioicismo e independentemente do uso de homeopatia - conforme as potências empregadas nesta pesquisa - o cultivo orgânico de Baccharis genistelloides subsp. crispa na primavera evidenciou que a resposta fenotípica é semelhante para a fitomassa correspondente a fenofase vegetativa das plantas, com poucas variações, bem como o teor de flavonóides totais, expresso em quercetina, demonstrando que a matéria prima vegetal apresenta potencial genético para melhoramento, bem como para futuros estudos em homeopatia vegetal desta espécie.

\section{REFERENCIA}

ARRUDA, V.M.; CUPERTINO, M.C.; LISBOA, S.P.; CASALI, V.W.D. Homeopatia tri-una na agronomia: as propostas de Roberto Costa e algumas relações com os agrosistemas. Viçosa: Fitotecnia - UFV. 2005. 119p.

ANDRADE, F.M.C.; CASALI, V.W.D.; DEVITA, B. CECON, P.R. BARBOSA, L.C.A. Efeito de homeopatias no crescimento e na produção de cumarina em chambá (Justicia pectoralis Jacq.). Revista Brasileira de Plantas Medicinais, v.4, n.1, p.19-28, 2001.

ANDRADE, F.M.C. Homeopatia no crescimento e na produção de cumarina em chambá - Justicia pectoralis Jacq. Viçosa, 2000, 214p. Dissertação (Mestrado em Fitotecnia), Universidade Federal de Viçosa.

BOFIM, F.P.G.; MARTINS, E.R.; DORES, R.G.R.;
BARBOSA, C.K.R.; CASALI, V.W.D.; HONÓRIO, I.C.G. Use of homeopathic Arnica montana for the issuance of roots of Rosmarinus officinalis L. and Lippia alba (Mill) N.E.Br. International Journal of High Diluition Research, v. 7, n. 23, p.113-117, 2008.

BONATO, C.M.; PROENÇA, G.T.; REIS, B. Homeopathic drugs Arsenicum álbum and Sulphur affect the growth and essential oil content in mint (Mentha arvensis L.). Acta Scientiarum Agronomy, v. 31, n. 1, p. 101-105, 2009.

BRANDÃO, M.; OLIVEIRA, A.K. Plantas ruderais e subespontâneas do Município de Ouro Preto e sua utilização na Medicina Popular. Daphne, v.5, n.2, p.4971, 1995.

BRASIL. Decreto $n^{\circ} 5.813$, de 22 de junho de 2006. Estabelece diretrizes e linhas prioritárias para o desenvolvimento de ações pelos diversos parceiros em torno de objetivos comuns voltados à garantia do acesso seguro e uso racional de plantas medicinais e fitoterápicos em nosso país, ao desenvolvimento de tecnologias e inovações, assim como ao fortalecimento das cadeias e dos arranjos produtivos, ao uso sustentável da biodiversidade brasileira e ao desenvolvimento do Complexo Produtivo da Saúde. Disponível em <http:// www.anvisa.gov.br> Acesso em: 05 fev. 2010.

BURG, C.I.; MAYER, H.P. Alternativas ecológicas para prevenção e controle de pragas e doenças. 17. ed. Francisco Beltrão: Editora Grafit, 2002.153p.

CAPORAL, F.J.M.; BOLDRINI, I.I. Florística e fitossociologia de um campo manejado na Serra do Sudeste, Rio Grande do Sul. Revista Brasileira de Biociências, v.5, n.2-3, p.37-44, 2007.

CARVALHO, L.M.; CASALI, V.W.D.; LISBOA, S.P.; SOUZA, M.A.; CECON, P.R. Efeito da homeopatia Arnica montana, nas potencias centesimais, sobre plantas de artemísia. Revista Brasileira de Plantas Medicinais, v.7, n.3, p.33-36, 2005.

CASALI, V.W.D.; ANDRADE, F.M.C. DUARTE, E.S.M. Acologia das Altas Diluições. Viçosa: UFV. 2009. 537p.

CASTRO, H.G.; FERREIRA, A.F. Contribuição ao estudo das plantas medicinais: carqueja (Baccharis genistelloides). Viçosa: UFV. 2000. 102p.

CLEMENT, C.R. 2001. Melhoramento de espécies nativas [Improvement of natives species]. In: Nass, L.L.; Valois, A.C.C.; Melo, I.S.; Valadares-Inglis, M.C. (Eds.). Recursos Genéticos \& Melhoramento - plantas. Fundação de Apoio à Pesquisa Agropecuária de Mato Grosso - Fundação MT, Rondonópolis, MT, pp. 423441. (Brasil)

CORRÊA Jr., C.; SCHEFFER, M.C. Boas práticas agrícolas (BPA) de plantas medicinais, aromáticas e condimentares. Curitiba: Instituto Paranaense de Assistência Técnica e Extensão Rural - EMATER. 2009. 52p.

FARMACOPÉIA BRASILEIRA, 4. ed. Fascículo II. São Paulo, 2002. p.182 (1-6).

GOBBO-NETO, L.; LOPES, N.P. Plantas Medicinais: Fatores de influência no conteúdo de metabólitos secundários. Química Nova, v.30, n.2, p.374-381, 2007.

GOTTLIEB, O.R.; KAPLAN, M.A.C.; BORIN, M.R.M.B. Biodiversidade: um enfoque químico-biológico. Rio de Janeiro: UFRJ. 1996. 267p.

KÖPEN, W.; GREIGER, R. Klimate der Erde. Gotha: Verlag 
Justus Perthes. 1928. (Mapa de parede 150x200cm).

LISBOA, S.P.; CUPERTINO, M.C.; ARRUDA, V.M.; CASALI, V.W.D. Nova visão dos organismos vivos e o equilíbrio pela homeopatia. Viçosa: Fitotecnia - UFV, 2005, 103p.

MACEDO, J.F. Fenologia da floração das plantas Invasoras no Campus-Pampulha da Universidade Federal de Minas Gerais. Daphne, v.5, n.4, p.15-27, 1995.

MONTANARI, R.M.; SOUSA, L.A.; LEITE, M.N.; COELHO, A.D.F.; VICCINI, L.F.; STEFANINI, M.B. Plasticidade fenotípica da morfologia externa de Lippia alba (Mill.) N. E. Br. Ex Britt \& Wilson (Verbenaceae) em resposta a níveis de luminosidade e adubação. Revista Brasileira de Plantas Medicinais, v.4, n.1, p.19-28, 2001.

PAVAN-FRUEHAUF, S. Plantas Medicinais de Mata Atlântica: manejo sustentado e amostragem. São Paulo: Annablume/Fapesp. 2000. 216p.

PIGLIUCCI, M. Evolution of phenotypic plasticity: where are we going now? TRENDS in Ecology and Evolution, v.20, n.9, p.481-486, 2006.

RITTER, M.R.; BAPTISTA, L.R.M. Levantamento florístico da família Asteraceae na "Casa de Pedra" e áreas adjacentes, Bagé, Rio Grande do Sul. Iheringia: Série Botânica, v.60, n.1, p.5-10, 2005.
RODRIGUES, V.E.G.; CARVALHO, D.A. Plantas medicinais no domínio do cerrados. Lavras: Editora da UFLA, 2001. 180p.

SAEG Sistema para Análises Estatísticas, Versão 9.1: Fundação Arthur Bernardes - UFV - Viçosa, 2007.

SCHILTING, C.D.; LEVIN, D.A. Phenotypic plasticity of annual Phox: tests of some hypothesis. American Journal of Botany, Columbus, v.71, n.2, p.252-260, fev. 1984.

SILVA, F.G. Estudos de calogênese in vitro e dos efeitos do manejo fitotécnico no crescimento e produção de óleo essencial em plantas de carqueja [Baccharis trimera (Less.) D.C.]. Lavras, 2001. 128f. Dissertação (Mestrado em Agronomia) - Universidade Federal de Lavras.

SOUSA, L.A. Produtividade e nutrição em três acessos de Baccharis trimera em função da adubação organo-mineral, em casa de vegetação. Botucatu, 2005. 42p. Dissertação (Mestrado em Horticultura), Universidade Estadual Paulista.

VIEIRA, R.F.; SILVA, S.R.; ALVES, R.B.N.; SILVA, D.B.; DIAS, T.A.B.; WETZEL, M.M.V.S.; UDRY, M.C.; MARTINS, R.C. eds. Estratégias para Conservação e Manejo de Recursos Genéticos de Plantas Medicinais e Aromáticas: Resultados da $1^{\circ}$ reunião técnica. Brasília: Embrapa/lbama/MMA/CNPq, 2002. 184p. 